\title{
The relationship between the thermal metamorphism of coal and the periodic law of the elements: a study based on the geochemical data of $C 2$ coal seam in the Fengfeng mining area of the Handan Coalfield in Hebei, China
}

\author{
JIANYE YANG ${ }^{1}$, BIN ZHENG $^{1}$
}

${ }^{1} X i$ ' an University of Science and Technology, Xi'an 710054, China Email: yjyxust@163.com

The periodic variation of the geochemical behavior of elements to the thermal metamorphism of coal was studied via using the differentiation mode and degree of elements in the thermal metamorphism process of the $\mathrm{C} 2$ coal seam, basing on the Fengfeng mining area of the Handan Coalfield in Hebei, China. This study confirms that the periodic variation of the elements geochemical behavior can be an indicator of the increasing of the metamorphism degree of a geological body. We obtained the coincidence degree (or similarity degree) between the geochemical behavior and the periodic variation of elements. Thus, there are two levels in the 57 elements: 1st-level elements, where the periodic variation of the geochemical behavior was largely synchronous with that of their first ionization energy, indicating single differentiation mode and inorganically associated elements; 2nd-level elements, where the differentiation mode deviates from their first ionization energy, and the large deviation will result in complicated and diverse differentiation mode. The rank of coal metamorphism affects on the proportion of elements with particular structural features among the 2nd-level elements, such as the $4 q+3$ type and the odd-odd elements. The elements distributions in organic and inorganic matter of coal are also closly related with the structural features of the elements. The differentiation mode could be characterized by the hierarchical parameter and differentiation intensity. They present positive or negative correlation with $R_{\max }$ in coal. The quantitatively ordered 57 elements in this study shows the goodness of fit and the correlation coefficient between $R_{\max }$ and the differentiation intensity. This study are consistent with the results of previous field research, which illustrates the scientific significance and application value of this study on the periodic variation of the geochemical behavior of elements. 